\title{
Synthesis of normal and variant human hypoxanthine-guanine phosphoribosyltransferase in Escherichia coli
}

(B-lymphoblast; crystallization; HPRT; PCR; recombinant DNA; x-ray diffraction analysis)

\author{
Beverly L. Davidson ${ }^{a}$, Jennifer E. Brown ${ }^{a}$, Christian H. Weber ${ }^{b}$, Thomas D. Palella ${ }^{a}$ and Blake \\ J. Roessler ${ }^{a}$
}

Departments of ${ }^{a}$ Internal Medicine or ${ }^{b}$ Biological Chemistry, University of Michigan Medical School, Ann Arbor, MI 48109, USA

Received by J.A. Engler: 26 May 1992; Accepted: 7 August 1992; Received at publishers: 11 September 1992

\section{SUMMARY}

Naturally occurring mutations in hypoxanthine-guanine phosphoribosyltransferase (HPRT) have been identified by amino acid sequencing, cDNA cloning, and direct nucleotide sequencing of PCR-amplified transcripts. To determine the effect these mutations have on the catalytic properties of the molecule, knowledge of the three-dimensional structure of HPRT is required. A prerequisite for this, however, is the availability of a large amount of purified product for crystallization and $x$-ray diffraction analysis. For these reasons we have developed an effective means of producing high levels of human HPRT in Escherichia coli using the expression cassette PCR. By taking advantage of a T7 polymerase/promoter system, we have expressed both normal and variant human hprt sequences in E. coli. The proteins synthesized from these sequences are immunologically and enzymatically active, and are physically indistinguishable from the HPRT in B-lymphoblasts derived from normal and three HPRT-deficient subjects.

\section{INTRODUCTION}

Hypoxanthine-guanine phosphoribosyltransferase (HPRT) is a purine salvage enzyme which catalyzes the formation of GMP and IMP from guanine and hypoxanthine, respectively, using PRPP as the phosphoribosyl donor. A partial deficiency of this cytosolic enzyme can

Correspondence to: Dr. B.L. Davidson, Department of Internal Medicine, University of Michigan Medical School, $1150 \mathrm{~W}$. Medical Center Dr., R5520 MSRB I, Ann Arbor, MI 48109-0680, USA. Tel. (313) 7473412; Fax (313) 763-4151; e-mail: Beverly.Davidson@med.umich.edu

Abbreviations: aa, amino acid(s); bp, base pair(s); DTT, dithiothreitol; ECPCR, expression cassette PCR; HPRT, hypoxanthine-guanine phosphoribosyltransferase; hprt, gene (DNA) encoding HPRT; IPTG, isothiopyranogalactoside; $\mathrm{kb}$, kilobase(s) or $1000 \mathrm{bp}$; $\mathrm{nt}$, nucleotide(s); PAGE, polyacrylamide-gel electrophoresis; PCR, polymerase chain reaction; PRPP, 5-phosphoribosyl-1-pyrophosphate; SD, Shine-Dalgarno; SDS, sodium dodecylsulfate; wt, wild type; [ ] denotes plasmidcarrier state. cause hyperuricemia, hyperuricaciduria, uric acid nephrolithiases, and in extreme cases, neurologic dysfunction (Kelley et al., 1967). Manifestations of complete deficiency, or the Lesch-Nyhan syndrome, include retarded growth, spasticity, choreathetoid movements, a tendency to self-mutilate and, in most cases, mental retardation (Lesch and Nyhan, 1964; Seegmiller et al., 1967). The pathophysiologic basis for these neurologic effects remains unclear.

The primary structure of human HPRT has been defined (Wilson et al., 1982b). The mature enzyme consists of 217 aa with the N-terminal Met removed and the penultimate Ala acetylated (Wilson et al., 1982b). Regions of $\alpha$-helix, $\beta$-sheet, and other secondary structure motifs have been predicted for the molecule based on this primary sequence information. Inferences on the tertiary structure of the molecule, with regards to PRPP and purine-binding sites, have been made based on comparisons of conserved structures in other PRTases and by defining 
mutations in kinetic variants (Hershey and Taylor, 1986; Argos et al., 1983). Based on these types of studies, aa $130-150$ and $140-210$ have been predicted to be involved in purine and PRPP binding, respectively. Both areas contain stretches of aa sequence which is highly conserved from $E$. coli to mammals. As noted, substitutions in these regions are usually deleterious to enzyme function.

The actual role of these putative binding domains, however, can only be addressed by $\mathrm{x}$-ray crystallographic analysis of normal and mutant enzymes, a major prerequisite of which is large amounts of purified protein. As demonstrated in this report, we have addressed this by developing a novel ECPCR method for cnhanced translation of human hprt sequences in E. coli. This technique was effective in producing high levels of both normal and mutant HPRT for subsequent purification.

\section{EXPERIMENTAL AND DISCUSSION}

\section{(a) Construction of an expression cassette}

The PCR primers used to generate the expression cassette for hprt were synthesized at the Oligonucleotide Synthesis Core Facility, University of Michigan. The 5' primer contains an EcoRI site, a T7 phage gene $10(\mathrm{Tn} 10)$ leader sequence (Olins et al., 1988), an SD consensus sequence, and 20 bp of normal human hprt coding sequence beginning with the A of the ATG start codon (Fig. 1). The $3^{\prime}$ primer consists of an EcoRI site $3^{\prime}$ of the terminal $15 \mathrm{bp}$ of HPRT-coding sequence (Fig. 1). Five $\mu \mathrm{g}$ of each primer, $100 \mathrm{ng}$ of hprt template in M13mp18, and $1 \mathrm{mM} \mathrm{dNTPs}$, werc combined in $1 \times T a q$ buffer $(2 \mathrm{mM} \mathrm{MgCl} / 10 \mathrm{mM}$ $\mathrm{KCl} / 10 \mathrm{mM}$ Tris $\mathrm{pH} 8.3 / 1 \mathrm{mM}$ DTT) and heated to $94^{\circ} \mathrm{C}$ for 3 min. After the addition of 1 unit Taq DNA polymerase (Ampli-Taq, Perkin Elmer/Cetus, Norwalk, CT) the mixture was subjected to five cycles of $94^{\circ} \mathrm{C}, 1 \mathrm{~min}, 22^{\circ} \mathrm{C}$, $1 \mathrm{~min}, 72^{\circ} \mathrm{C}, 2 \mathrm{~min}$ and then 30 cycles of $94^{\circ} \mathrm{C}, 1 \mathrm{~min}$, $72^{\circ} \mathrm{C}, 2 \mathrm{~min}$. The PCR reaction products were cut with EcoRI (Bethesda Research Laboratories) and purified on a Qiagen ${ }^{\mathrm{TM}}$ tip-5. The hprt expression cassettes for mutant sequences were made by performing the PCR on cDNAs generated by reverse transcribing $1 \mu \mathrm{g}$ total RNA isolated from B-lymphoblast cell lines derived from patients K.C., P.C., and D.B., using previously described techniques
(Table I; Davidson et al., 1989b). Conditions for amplifying and purifying mutant sequences were the same as those used for the wt template.

Expression cassettes for normal and mutant sequences were cloned into EcoRI-cut pSP72 (Promega, Madison, WI) downstream from the $\mathrm{T} 7$ promoter, and used to transform E. coli DH $5 \alpha$ (Bethesda Research Laboratories). All expression plasmids were subjected to dideoxynucleotide sequencing to insure authenticity of sequence. The wt clone pSPSDE3, and variant clones pSPPC24, pSPKC4 and pSPDB1 (representing HPRT $_{\text {Ashville, }}$ HPRT $_{\text {Ann Arbor }}$, and HPRT London, $_{\text {, respectively) were then }}$ used to transform BL21(DE3) for high-level production of human HPRT (Olins et al., 1988). The plasmids arc described in detail in Table II.

\section{(b) Time course of production of human HPRT in Escherichia coli}

Fig. 2 is an SDS-PAGE gel of aliquots from a time course of induction of BL21(DE3)[pHPSDE3]. The production of a $24-\mathrm{kDa}$ protein is evident in as little as 30 min post induction, and continues for up to $8 \mathrm{~h}$ (lanes $2-7$ ). When the induction is allowed to continue for $24 \mathrm{~h}$, there is little change in the relative amount of protein produced as compared to the $8 \mathrm{~h}$ time point (data not shown). As seen in lanes 8 and 9 , there is no concomittant increase in the expression of a $24-\mathrm{kDa}$ protein when pSP72 in BL21(DE3) is used; only the T7 polymerase subunits are induced. Note that expression of these subunits in the HPRT-expressing plasmids appears to be repressed, presumably due to the enhanced translation.

Immunoblotting was done using anti-human HPRT antibody to identify the overexpressed $24-\mathrm{kDa}$ protein. As evident in lane 1 of Fig. 3, low levels of human HPRT are expressed before the addition of IPTG. This is presumably due to the fact that the lacUV5 promoter driving transcription of the 17 polymerase gene is not tightly repressed, even in the absence of IPTG. However, when IPTG is added, there is a large increase in the level of HPRT expressed by pHPSDE3 (lanes 2-4). Human HPRT is also evident in lane 6, which is BL21(DE3)[pHPSDE5]. This construct contains human hprt cloned in the opposite orientation to the $\mathrm{T} 7$ promoter. In this context, low-level synthesis of HPRT may occur as a result of transcription off an upstream pro-

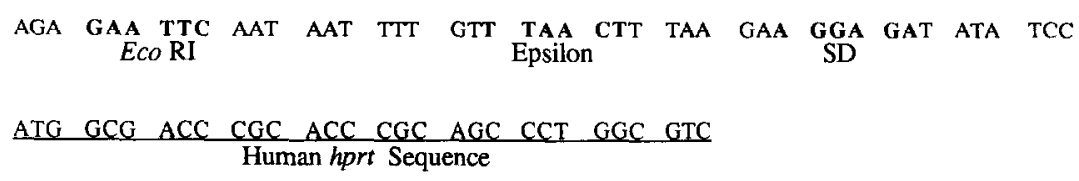

Fig. 1. Expression cassette for expressing human hprt in E. coli. The cassette contains an EcoRI cloning site, the $\varepsilon$ (epsilon) sequence from $\mathbf{T} 7$ bacteriophage gene 10 (Olins et al., 1988), and an SD sequence. The sequences which overlap with human hprt cDNA sequences are indicated (bold). 
TABLE I

HPRT: aa substitutions and physical properties of HPRT variants

\begin{tabular}{lllll}
\hline Cell line & Variant & The aa substitutions & Physical properties & Ref $^{\mathrm{a}}$ \\
\hline D.B. & London & $\mathrm{Ser}^{110} \rightarrow$ Leu & increased anodal migration on SDS-PAGE & A \\
K.C. & Ann Arbor & $\mathrm{Ile}^{132} \rightarrow \mathrm{Met}$ & normal migration on SDS-PAGE and nondenaturing PAGE & B \\
P.C. & Ashville & Asp $^{201} \rightarrow$ Gly & decreased anodal migration on nondenaturing PAGE & C \\
\hline
\end{tabular}

a A, Davidson et al. (1988); B, Fujimon et al. (1988); C, Davidson ct al. (1989a).

TABLE 11

Plasmids of variant hprt cDNAs

\begin{tabular}{lll}
\hline Variant $^{\mathrm{a}}$ & The nt substitution $^{\mathrm{b}}$ & Plasmid $^{\mathrm{c}}$ \\
\hline wt & & pSPSDE3 \\
D.B. & $\mathrm{C}^{329} \rightarrow \mathrm{T}$ & pSPDB1 \\
K.C. & $\mathrm{T}^{399} \rightarrow \mathrm{G}$ & pSPKC4 \\
P.C. & $\mathrm{A}^{602} \rightarrow \mathrm{G}$ & pSPPC24
\end{tabular}

${ }^{\text {a }}$ See Table 1 for details regarding variants.

bSubstitution of $\mathrm{nt}$ with respect to wt sequence.

'The plasmid backbone is pSP72 (Promega, Madison, WI).

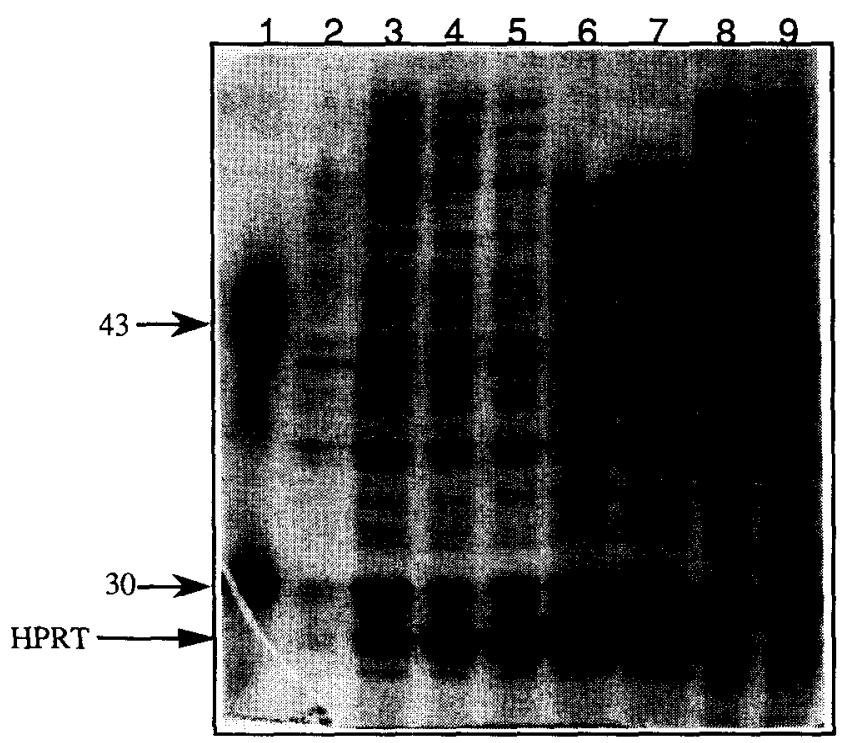

Fig. 2. Time course of synthesis of human HPRT in E. coli. Human HPRT was produced by the addition of $0.4 \mathrm{mM}$ IPTG to cultures of BL21(DE3)[pHPSDE3] when $A_{600}$ reached 0.4. Aliquots $(1 \mathrm{ml})$ were removed at $0,30 \mathrm{~min}, 1,2,4$, and $8 \mathrm{~h}$ (lanes 2-7, respectively), centrifuged $3 \mathrm{~min}, 14000 \times \mathrm{g}$, washed in $1 \mathrm{ml}$ Hanks Balanced Salt Solution (HBSS, Gibco) divided into two $500 \mathrm{ml}$ aliquots and centrifuged again for $3 \mathrm{~min}$ at $14000 \times \mathrm{g}$. One cell pellet was resuspended in Laemmli SDS-PAGE buffer and a portion analyzed by $0.1 \%$ SDS- $12 \%$ PAGE. Note the rapid expression of human HPRT (large arrow). IPTG was also added to cultures of BL21(DE3)[pSP72]. These lysates were analyzed 1 and $8 \mathrm{~h}$ post induction (lanes 8 and 9 ). Lane $1, M_{\mathrm{r}}$ markers. Molecular size (in $\mathrm{kDa}$ ) are indicated on the left margin.

moter (for example $\beta$-lactamase) in conjunction with the strong translational enhancer sequences. In the parent plasmid alone (lane 6) no HPRT is seen even $8 \mathrm{~h}$ postinduction.

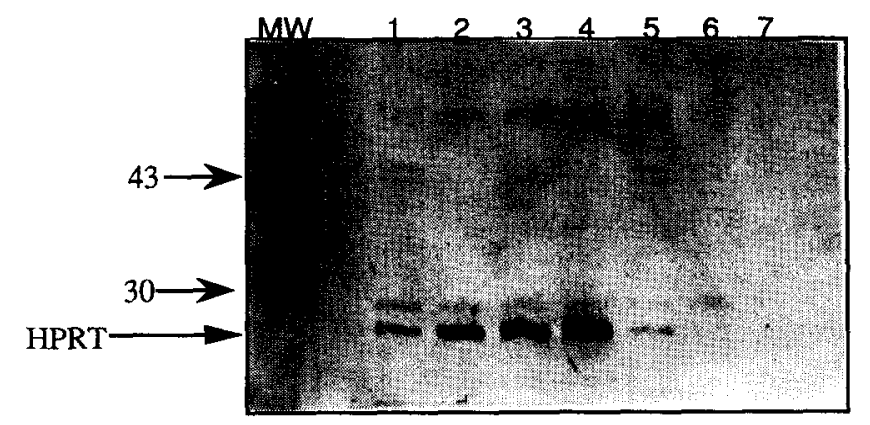

Fig. 3. Immunoblot of human HPRT produced in E. coli. Cultures of BL21(DE3)[pHPSDE3] were analyzed for the presence of human HPRT at $0,30 \mathrm{~min}, 1$, and $2 \mathrm{~h}$ post induction (lanes 1-4). Protein was blotted onto PVDF membranes (Millipore), blocked $2 \mathrm{~h}$ at room temperature with TNS $/ 0.5 \%$ Tween-20 (TT buffer; TNS is $20 \mathrm{mM}$ Tris pH $7.5 / 500 \mathrm{mM} \mathrm{NaCl}$ ), washed (all washes and antibody dilutions were done in TT buffer unless otherwise indicated), incubated overnight with anti-human HPRT antibody at $4^{\circ} \mathrm{C}$, washed, incubated with goat-antirabbit antibody conjugated to alkaline phosphatase (Kirkegaard and Perry Laboratories, Inc., Gaithersburg, MD) for $2 \mathrm{~h}$ at room temperature, washed in TNS, and incubated with NBT/TIBS substrate (Kirkegaard and Perry Laboratories). Lane 5 is of BL21(DE3)[pSPSDE5] lysate in which the hprt cDNA is cloned antisense to the T7 promoter in pSP72. Lane 6 is lysate from BL21(DE3)[pSP72]. Cultures of BL21(DE3)[pSPSDE5] and BL21(DE3)[PSP72] were harvested $4 \mathrm{~h}$ after induction. Lane 7 contains $4 \mathrm{ng}$ of purified human HPRT, the lower limit for this detection system. Gel as in Fig. 2. MW, prestained molecular size markers (Amersham). Molecular sizes as in Fig. 2. Large arrow points to the human HPRT.

\section{(c) Purification of human HPRT from Escherichia coli}

Large-scale cultures (500-ml aliquots) of BL21(DE3)[pHPSDE3] were grown, and the cells harvested and lysed as described. Xanthine was added to a concentration of $1 \mathrm{mM}$ before column loading to reduce the binding of $E$. coli xpt/gpt to the affinity sites. Bound human HPRT was eluted with buffer A containing $1 \mathrm{mM} \mathrm{GMP,}$ and the fractions containing the peak of human HPRT collected and concentrated. As seen in the SDS-PAGE gel in Fig. 4 (lane 2), the eluate contains more than $95 \%$ pure human HPRT. Initially, a molecular sieve column was included in the purification scheme, and was run before the affinity column. However, this did not significantly increase the purity of the final product (data not shown).

\section{(d) Production of mutant HPRT in Escherichia coli}

cDNAs were generated from threc previously characterized human HPRT variants, HPRT $_{\text {Ann }}$ Arbor, 


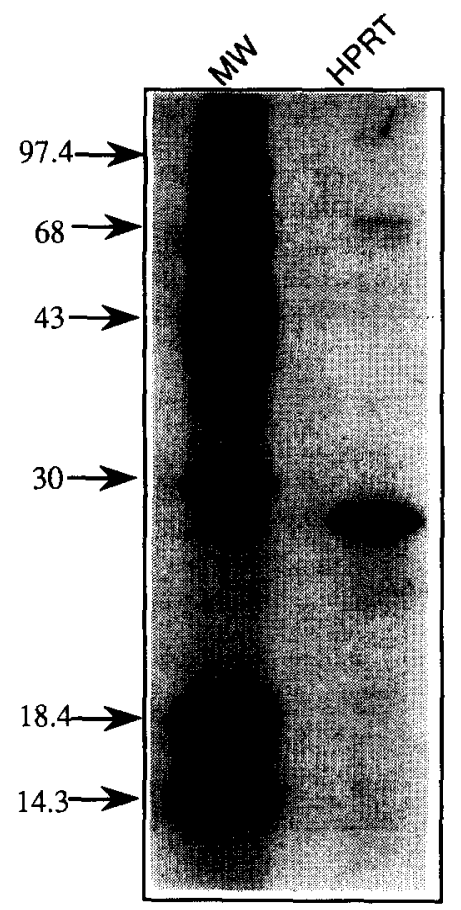

Fig. 4. Purified human HPRT isolated from E. coli. For purification, single colonies were placed in $3 \mathrm{ml}$ of $\mathrm{LB}$ broth containing $30 \mu \mathrm{g}$ ampicillin/ml, allowed to grow to $A_{600}$ of $0.4-0.6$, and transferred to $500 \mathrm{ml}$ LB broth. The culture was allowed to reach $A_{600}$ of 0.4 , after which IPTG was added to $0.4 \mathrm{mM}$ final concentration. Inductions ranged from $4 \mathrm{~h}$ to overnight for large scale cultures. After induction the cells were harvested, washed in buffer $\mathrm{A}$, and resuspended in buffer $\mathrm{A}$ containing $10 \%$ glycerol. The suspension was lysed by French press, debris pelleted, and the remaining lysate brought to $1 \mathrm{mM}$ xanthine. The lysate was then passed over a GMP-agarose affinity column (Sigma Biochemicals). The column was washed with buffer A (buffer A is $50 \mathrm{mM}$ Tris $\mathrm{pH} 7.4 / 25 \mathrm{mM} \mathrm{KCl} / 10 \mathrm{mM} \mathrm{MgCl}_{2} / 1 \mathrm{mM}$ DTT), and human HPRT eluted with $1 \mathrm{mM}$ GMP in buffer $\mathrm{A}$. Those fractions containing human HPRT were pooled, concentrated, and analyzed for purity by $0.1 \%$ SDS- $12 \%$ PAGE followed by Coomassie staining. Molecular size in $\mathrm{kDa}$ are indicated on the left margin.

HPRT $_{\text {Ashville, and }} \mathrm{HPRT}_{\text {London }}$ (Table I), by reverse transcribing RNA isolated from B-lymphoblasts derived from patients K.C., P.C., and D.B., respectively, followed by ECPCR. The resultant expression cassettes were then cloned into pSP72 and transformed into BL21(DE3) as described. Colonies were induced for production of HPRT, and analyzed by SDS-PAGE and nondenaturing PAGE followed by activity gel assay. As noted in Table I, HPRT $_{\text {London }}$ migrates more anodally than does normal HPRT in SDS-PAGE of B-lymphoblast lysates. This physical property is reproduced in pHPDB1, the expression cassette representing this variant (Fig. 5A). Immunoblotting confirmed that the faster migrating species was HPRT (Fig. 5B). The HPRT produced from pSDKC4 and pSDPC21 exhibited normal migration on SDSPAGE following induction with IPTG, consistent with the corresponding variant protein from B-lymphoblasts (data not shown).

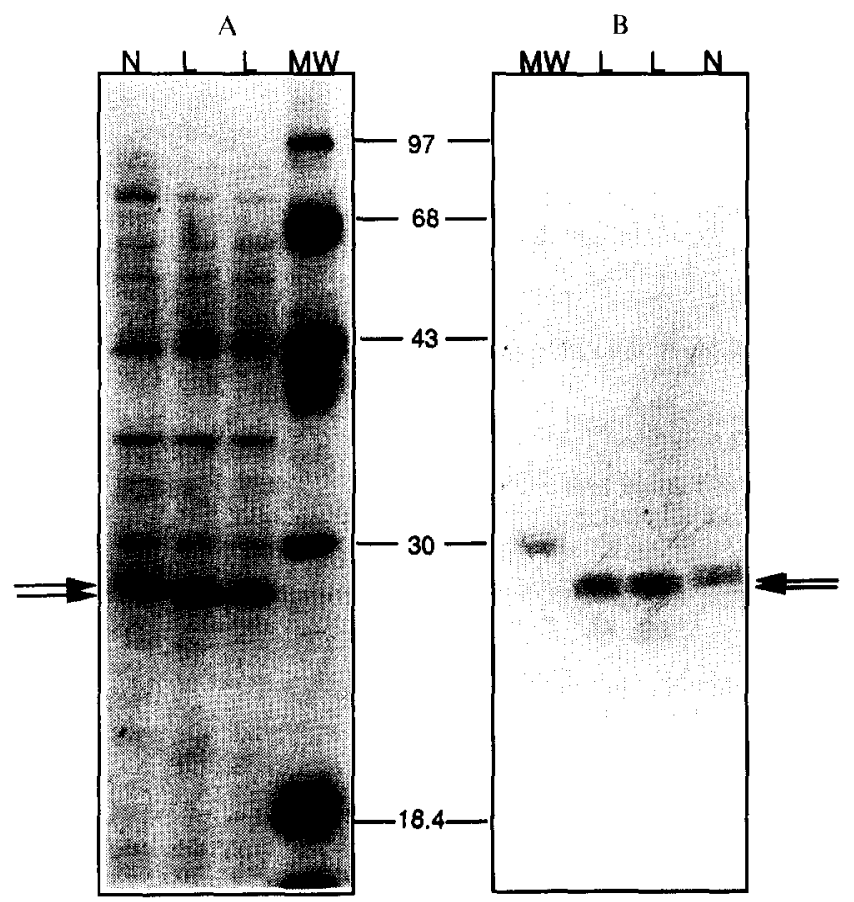

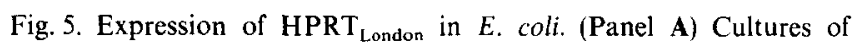
BL21(DE3)[pSPDB1] and BL21(DE3)[pSPSDE3] were induced, and lysates analyzed by SDS-PAGE (as in Fig. 2). The arrows denote the differences in migration between normal (N) and London (L) HPRTs. $\mathrm{MW}$, rainbow markers (Amersham). Molecular sizes in $\mathrm{kDa}$ are indicated. (Panel B) Immunoblot of the SDS polyacrylamide gel in panel $\mathbf{A}$.

Lysates from induced cultures of pSDDB1, pSDKC4 and $\mathrm{pSDPC} 21$ were also run on native gels and then assayed in situ for HPRT activity. As expected, protein synthesized from pSDKC4 had normal migration whilc pSDPC21 was less anodal than normal (Fig. 6). Again, these properties are consistent with those of the B-lymphoblast proteins.

The use of the $\mathrm{T} 7$ bacteriophage gene 10 translational enhancer sequences upstream from the required SD sequence in the expression cassette greatly improved the expression of $\mathrm{T} 7$ promoter-driven sequences (Fig. 1). When expression cassettes were made containing only the normal hprt cDNA construct plus SD sequences, the production of HPRT was minimal (data not shown). Indeed, the presence of human HPRT was visualized only by nondenatured PAGE/activity assay in lysates from these cultures, and not by SDS PAGE. Additionally, we saw only variable levels of low expression when this cassette was cloned downstream from a heat-inducible tac promoter (data not shown). However, the tac promoter system was not tried in conjunction with the strong translational enhancer sequences.

An additional consideration when using strong translational enhancer sequences is the 'leakiness' of the promoter driving T7 polymcrase. As evident in Fig. 3, human HPRT was synthesized, albeit at low levels, even in the 


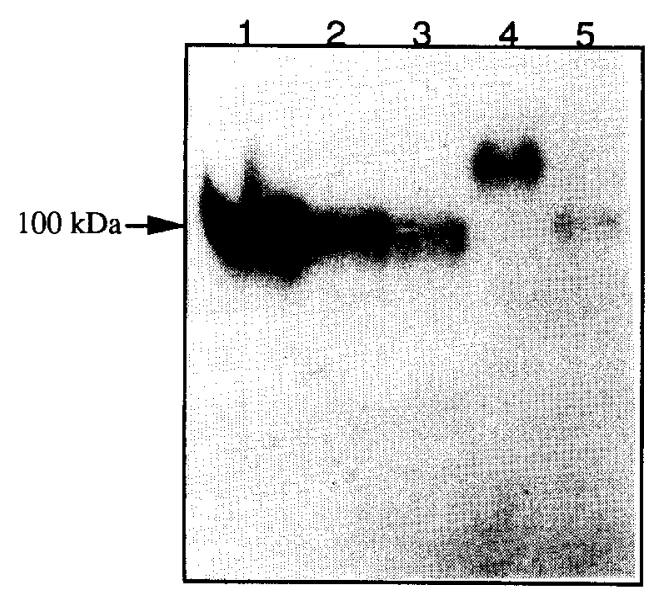

Fig. 6. Analysis of synthesised proteins by native 6\% PAGE and activity assay. Cultures containing the variant hprt expression cassettes were induced for $4 \mathrm{~h}$, harvested, and aliquots resuspended in lysis buffer (20 $\mu \mathrm{g}$ lysozyme/ml in $10 \mathrm{ml} \mathrm{MgCl} / 20 \mathrm{mM} \mathrm{KCl} / 50 \mathrm{mM}$ Tris $\mathrm{pH} 7.4$ ), incubated for $30 \mathrm{~min}$ at room temperature, and centrifuged at $14000 \times \mathrm{g}$ for $5 \mathrm{~min}$. Non-denatured lysates were run on $6 \%$ polyacrylamide gels and assayed in situ for human HPRT activity using previously described techniques (Davidson et al,, 1989a). Lanes: 1, $5 \mu \mathrm{g}$ of protein from GM558 lysate, a B-lymphoblast line derived from a normal male; 2-5, E. coli BL21(DE3) cells carrying: pSPDB1, pSPKC4, pSPPC24, and pSPSDE3, respectively (described in Table II).

absence of IPTG. If the gene of interest is deleterious to cell viability such an enhancer may be counterproductive. Based on this, a variety of combinations of translation enhancer/promoter systems may need to be tried to maximize protein production.

The one-step purification scheme for obtaining human HPRT is a dramatic improvement over that required to isolate purified human HPRT from erythrocyte lysates (Wilson et al., 1982b), making this an attractive alternative for obtaining large amounts of the purified enzyme for crystallographic analysis. Furthermore, one of the variant proteins chosen for this study, HPRT London, has been purified in small amounts from erythrocytes using a GMP-affinity column (Wilson et al., 1983). Crystallization and analysis of these two proteins alone will enable us to understand the elevated (sixfold) $\mathrm{K}_{\mathrm{m}}$ for hypoxanthine in this variant.

\section{(e) Conclusions}

ECPCR done with expression cassettes containing strong translational enhancer sequences enabled us to synthesize and purify active human HPRT in $E$. coli. In addition, variant sequences were expressed, with the resultant proteins exhibiting physical properties indistinguishable from their B-lymphoblast counterparts. These results complete an important preliminary step towards understanding the enzymology of HPRT, namely, producing large quantities of the protein for crystal growth, diffraction analysis, and three-dimensional structure determination.

\section{ACKNOWLEDGEMENTS}

The authors wish to thank Mary J. Maliarik for many helpful discussions, and Jeanne-Marie Quevedo for excellent secretarial support. B.L.D. and B.J.R. are fellows of the National Arthritis Foundation.

\section{REFERENCES}

Ausubel, F.M., Brent, R., Kingston, R.E., Moore, D.D., Seidman, J.G., Smith J.A. and Struhl, K.: Current Protocols in Molecular Biology. Wiley, New York, 1989.

Argos, P., Hanei, M., Wilson, J.M. and Kelley, W.N.: A possible nucleotide-binding domain in the tertiary fold of phosphoribosyltransferases. J. Biol. Chem. 258 (1983) 6450-6457.

Davidson, B.L., Chin, S.J., Wilson, J.M., Kelley, W.N. and Palella, T.D.: Hypoxanthine-guanine phosphoribosyltransferase. Genetic evidence for identical mutations in two partially deficient subjects. J. Clin. Invest. 82 (1988) 2164-2167.

Davidson, B.L., Pashmforoush, M., Kelley, W.N. and Palella, T.D.: Human hypoxanthine-guanine phosphoribosyltransferase deficiency. The molecular defect in a patient with gout (HPRT Ashville $)$. J. Biol. Chem. 264 (1989a) 520-525.

Davidson, B.L., Tarle, S.A., Palella, T.D. and Kelley, W.N.: Molecular basis of hypoxanthine-guanine phosphoribosyltransferase deficiency in ten subjects determined by direct sequencing of amplified transcripts. J. Clin. Invest. 84 (1989b) 342-346.

Fujimori, S., Hidaka, Y., Davidson, B.L., Palella, T.D. and Kelley, W.N.: Identification of a single nucleotide change in a mutant gene for hypoxanthine-guanine phosphoribosyltransferase (HPRT Ann Arbor $_{\text {) }}$. Hum. Genet. 79 (1988) 39-43.

Hershey, H.V. and Taylor, M.W.: Nucleotide sequence and deduced amino acid sequence of Escherichia coli adenine phosphoribosyltransferase and comparison with other analogous enzymes. Gene 43 (1986) 287-293.

Kelley, W.N., Rosenbloom, F.M., Henderson, J.F. and Seegmiller, J.E.: A specific enzyme defect in gout associated with overproduction of uric acid. Proc. Natl. Acad. Sci. USA 57 (1967) 1735-1739.

Lesch, M. and Nyhan, W.L.: A familial disorder of uric acid metabolism and central nervous system function. Am. J. Med. 36 (1964) 561.

Olins, P.O., Devine, C.S., Rangwala, S.H. and Kavka, K.S.: The T7 phage gene 10 leader RNA, a ribosome-binding site that dramatically enhances the expression of foreign genes in Escherichia coli. Gene 73 (1988) 227-235.

Seegmiller, J.E., Rosenbloom, F.M. and Kelley, W.N.: Enzyme defect associated with a sex-linked human neurological disorder and excessive purinc synthesis. Science 155 (1967) 1682-1684.

Wilson, J.M., Baugher, B.W., Mattes, P.M., Daddona, P.E. and Kelley, W.N.: Human hypoxanthine-guanine phosphoribosyltransferase. Demonstration of structural variants in lymphoblastoid cells derived from patients with a deficiency of the enzyme. J. Clin. Invest. 69 (1982a) 706-715.

Wilson, J.M., Tarr, G.E., Mahoney, W.C. and Kelley, W.N.: Human hypoxanthine-guanine phosphoribosyltransferase. Complete amino acid sequence of the erythrocyte enzyme. J. Biol. Chem. 257 (1982b) 10978-10985.

Wilson, J.M., Tarr, G.E. and Kelley, W.N.: Human hypoxanthine (guanine) phosphoribosyltransferase: an amino acid substitution in a mutant form of the enzyme isolated from a patient with gout. Proc. Natl. Acad. Sci. USA 80 (1983) 870-873. 